\title{
Modeling and Simulation of a New Integrated Electrohydraulic Actuator for Humanoid Robots
}

\author{
Samer Alfayad ${ }^{1 *}$, Elmira Amrollah ${ }^{2}$, Abdellatif A ${ }^{1}$, Fethi B Ouezdou ${ }^{1}$, Salem A \\ Haggag $^{3}$, Faycal Namoun ${ }^{2}$ \\ ${ }^{1}$ Laboratoire d'Ingénierie des Systèmes de Versailles (LISV), EA4048 Université de Versailles Saint-Quentin-en-Yvelines, Paris-Saclay University, Vélizy, \\ France. \\ ${ }^{2}$ BIA ZA Les Boutriers, 8 rue de l'Hautil, 78000 Conflans fin d'Oise, France. \\ ${ }^{3}$ School of Engineering, Department of Mechanical Engineering, P. O. Box 28282, Dubai, United Arab Emirates.
}

\begin{abstract}
Received: October 21, 2016; Accepted: October 25, 2016; Published: November 10, 2016
*Corresponding author: Samer Alfayad, Laboratoire d'Ingénierie des Systèmes de Versailles (LISV), EA4048 Université de Versailles SaintQuentin-en-Yvelines, Paris-Saclay Université, Vélizy, France. E-mail: samer.alfayad@lisv.uvsq.fr
\end{abstract}

\begin{abstract}
The work presented in this paper is an important step toward a better understanding of a compact hydraulic robotic actuator, based on the Integrated Electro-Hydraulic Actuator (IEHA) developed by Alfayad and Ouezdou [1]. The novel advantage of this actuator is being highly compact and autonomous (no need for central hydraulic source), while keeping a good power to weight ratio. In order to present and develop the working dynamics of this actuator, a highly detailed mathematical model for the system is presented. The proposed model is simulated using MATLAB-Simulink software to identify the effect of the internal system parameters on system dynamics and prepare an input-output test-bed model. Such test-bed model is used to obtain the transfer function of the system and its order. Analysis of the effects of the main parameters was carried out and a lower order of the system was identified. A linear model of the system is derived and validated using system identification technique. Finally, a robust motion controller is applied on the proposed linear model and the simulation results are presented.
\end{abstract}

Keywords: Hydraulic Actuation; Mathematical Modeling; System Identification Technique; Virtual Modeling;

\section{Nomenclature}

Cd: The venta contracta coefficient [-]CHeA \& CHeB The two sides of the carriage moving w.r.t the fixed frame [-]; d: Distance between the bottom of chamber and The center of the shaft [mm]; E: Eccentricity of the integrated actuator [mm]; Fp: The force exerted by the oil pressure in the Radial pump on the carriage [N]; Fe: The hydraulic force in the carriage chambers [N]; Fext: The external force exerted on the cylinder [N]; Fc: The hydraulic force exerted on the cylinder [N]; Hp1: The distance between the pistons of length l_p at the dead bottom position and the bottom of its chamber [mm]; Hp2: The distance between the pistons at the high Dead point and the bottom of its chamber [mm]; Hpi: The height of oil in piston chamber (i) at each Instant [mm]; Kin: Internal leakage coefficient [-]; Kout: Internal leakage coefficient [-]; Lp: The stroke length of the piston [mm]; Lpi:
The distance between the shaft center + Piston contact point on the surface of the Housing [mm]; m: The end effectors mass [kg]; me: Mass of the carriage of the IEHA [kg]; N: Number of micropistons [-]; Prp: The pressure of the oil at the intake channel of the radial pump [bar]; Pc: The pressure difference between the two Cylinder chambers A and B [bar]; Ps: High pressure line [bar]; PA: Pressure in chamber A of the micro-pistons [bar]; PB: Pressure in chamber B of the micro-pistons [bar]; Pi: The micropistons of the IEHA [-]; Qmac: The average macroscopic flow of the N Micro-pistons [m3/s]; Qmic: The average microscopic flow of the N Micro-pistons [m3/s]; Qe: The flow from microvalve into the carriage Chambers [m3/s]; Qeleak: Leakage flow from the carriage micro-Pistons to the body of the actuator [m3/s]; Q: The flow from the micro-pump [m3/s]; QleakA: The leakage between the carriage and output Cylinder + the one between the hydraulic Chamber to the micro pumps body [m3/s]; Qpileak: The leakage flow of the micro-pistons in the Radial pump [m3/s]; Qleak: The internal leakage between the two Chambers of the cylinder [m3/s]; $\mathbf{R b}$ : The radius of the carriage $[\mathrm{mm}]$; rtig: The micro-valve radius $[\mathrm{mm}]$; rrp: The radius of the in-out opening section of the micro-pump [mm]; Sc: The surface area of the linear hydraulic Cylinder's piston [cm2]; Se: The surface area of the carriage chambers [cm2]; Spi: The active area of a single piston [mm2]; V: Fluid volume [m3]; ve: The volume of the chamber of the carriage [m3]; vpi: The volume of the IEHA micro-pistons [m3]; vc: The volume of the chamber of the output Cylinder [m3]; X: Micro-valve input displacement [mm]; Y: The end effector (output load cylinder) Position [mm]; $\boldsymbol{\beta}$ : Bulk modulus of elasticity [MPa]; $\boldsymbol{\theta}$ : The angle between the piston and the Reference axe [degrees]; $\boldsymbol{\omega}$ : Rotational speed of the shaft [rad/sec]; $\boldsymbol{\rho}$ : The density of the oil $[\mathrm{kg} / \mathrm{m} 3]$; $\boldsymbol{\zeta}$ : The actual pressure in the piston chamber [bar]; $\boldsymbol{\varphi}$ i: Phase angle of the piston pi [deg]

\section{Introduction}

The development of bio-inspired robots; an important and 
active area of research; has been going on for the past few years. These robots outperform the mobile robots in terms of mobility and versatility [2]. Moreover, they combine many desirable features such as human-like locomotion capabilities and humanfriendly design and behavior. However, the performances exhibited by bio-inspired robots are more or less limited. This is mainly due to their actuation. Indeed, in our opinion, the first still an open question that should be answered while designing a bio-inspired robot concerns its actuation.

Research in the actuation of bio-inspired robots that aims to mimic the performances of biological systems, has been investigated by several research teams. Nevertheless, as far as we know, no actuator able to reproduce the biological muscle capabilities in term of producing force and speed already exists. Focusing on humanoid robots, a continuous need for enhancing their performances leads to identifying the desired actuator properties. These properties are: i) high power to mass ratio; ii) high integration within the robot body; iii) safe-interaction of the humanoid with the surrounding environment while performing human-like behavior.

Basically, the actuation for humanoid robots can be either electrically or hydraulically. Most robotic applications are electrically driven. Generally, electric motors with high gear ratio drives are popular because of their small size and cheap price. In addition, electric motors are proven to be easy to use and control. Significant examples of electrically actuated robots are: ASIMO [3], ROBIAN [4], HRP biped series [5], Johnnie and LOLA [6], REEM [7].

However, electric actuation has several drawbacks. Indeed, electric motors normally produce small torques relative to their size and weight, thereby making reduction sub-systems with high ratios essential to convert velocity into torque. These reduction components are limited and cannot increase indefinitely, which resulted in having reduced dynamic capabilities systems. Moreover, the presence of high reduction ratios causes limited passive back-drivability, which may lead to unsafe interaction with humans as well as troubles for walking in unforeseen terrains [8].

Nevertheless, several research works to enhance the performance of electric actuators for humanoid robots were proposed. For instance, flexible elements were added between the motor and the load, as can be seen in ECD leg [9], Tekken [10] in order to have locomotion activities over unforeseen terrain. Other robots, like COG [11] and DOMO use series elastic actuators developed by Robinson et al [12]. In this kind of robots, robustness of the system is an issue because of complex electric connection [13]. On the other hand, harmonic drives were also utilized for example in ARMAR III [14]. In this robot, motors and the harmonic drives are located in the thorax of the robot. This design intended to decrease the weight of the arm. However, this inherently leads to complex transmission system through wires, which also reduces the robustness.

In general, electric actuators coupled with gearbox reduction systems currently didn't fulfill all the needs of humanoid robots.
Indeed, this actuation solution neither has a high power-toweight ratio nor is able to simultaneously provide the speed and forces required for highly dynamic robots. Therefore, other methods of actuation are commonly sought after.

The other concurrent actuation solution is hydraulic technology based. It has several advantages, which mainly include: 1) high power to mass ratio; 2) ability to produce high torque at low speed; 3 ) the high stiffness compared to electric ones; 4) the ability to perform continuous, intermittent, reversing and stalled motions without damage; 5) the ability to emulate human musclo-skeletal systems by means of high-bandwidth force control. Examples of robots using hydraulic actuation include: Bigdog [15], Sarcos [16], HyQ [17], Tae-Mu [18] and the underdevelopment humanoid robot HYDRÖ̈D [19].

However, this type of actuators has its main drawbacks. The major one is due to the necessity of a central Hydraulic Power Unit (HPU) to supply high-pressure fluid to all the robot joints. . This HPU is always bulky and the leakage from the hydraulic tube connections can cause safety issues, especially in human-robot interaction. Hence, it was necessary to have integrated hydraulic actuators inside the robot and near the joints.

Research for hydraulic actuator for robotics has been started with Bobrow et al [20], in which a closed loop hydrostatic actuator was introduced. This actuator was driven directly from an electric motor without a gear train enabling large speed reductions and corresponding torque amplification. However, achieving high torques is not possible without the use of large electric motors and power amplifiers, which leads to an increased overall dimensions and large mass for the system. Moreover, this actuator suffered from dead band caused by the inversion of electric motor rotation. S. Habibi et al. followed [21], with an improvised electrohydraulic actuator that tackled the effect of dead band by using a high gain cascaded control strategy with motor speed feedback. Nevertheless, this actuator had several design constraints to achieve high performance. These constraints include the usage of a symmetrical actuator in addition to appropriate sizing of hydraulic components to minimize the pressure drop. These constraints questioned the supposed compactness of an electrohydraulic actuator dedicated for robotic applications.

Recent research was done on the EHA compactness by Gnesi et al [22] and Takahashi et al [23], in which both presented two EHAs made for aircraft applications. The first presented an EHA with a vane pump and double acting cylinder, while the latter used a similar design but with a piston pump. The most recent contribution was attributed to Altare et al [24] and [25], in which a miniature gear pump is presented along with its actuator. The volumetric displacement of this EHA was found to be $0.13 \mathrm{cc} / \mathrm{rev}$ and it was also, made solely for aerospace applications.

In 2011, an IEHA (Integrated Electro-Hydraulic Actuator) was developed by Alfayad et al. [26] and [31]. Its main objective was eliminating the need for a central pressure source and to be implemented for each joint of the humanoid hydraulic robot HYDROïD. Due to the different pressures needed by the robot 
joints, each single IEAH actuator can be considered autonomous. Thanks to its compactness, the IEHA can be placed as near as possible to the hydraulic actuator (rotary or linear). Hence, the pressure drop is reduced and leakage may only exist inside the actuator. Moreover, the IEHA contains a passive distributor connecting the pump with the actuator. This passive distributor delivers the oil between the pump and the actuator without the need of flipping the direction of the electric motor driving the pump. Hence, the effect of dead band is avoided enhancing the position control of the actuator. In addition to the dead band elimination, the presence of the passive distributor enables the IEHA to handle any asymmetrical actuator. Therefore, we believe that this type of actuation is a one of the best choices for enhancing the capabilities of robotic systems.

The main objective for the IEHA is to be implemented on the humanoid robot HYDROïD. Hence, the human robot interaction and compliance are of high priority. There are different ways to ensure that the robot does not risk injuring the user. One approach to soft human robot contact is back-drivability. This ability enables the mechanical system to move the input axis from the output axis. In other words, the force applied from output axis of the actuator must be greater than force lost in the actuator due to static friction $[27,28]$. A more advanced approach is to apply an active compliance, which can be reached through accurate force/position control [29]. Both of these approaches need a complete dynamic model of the actuation system, including the IEHA actuators and the several transmission mechanisms.

Consequently, the goal of the work presented in this paper concerns the study the internal parameters of the IEHA which highly complex and therefore identify their influence on the behavior of the whole system. The ultimate goal is deducing a practical dynamic model that approximates the IEHA behavior for active compliance control targeting. Indeed, the dynamic model will be used to choose the best control strategy to ensure the back-drivability. Moreover, the identification of the internal parameters in the literature was done in most cases through empirical assumptions and experimental results. Due to the high compactness of this kind of actuators. In the presented contribution, the approach is based on the identification of the IEHA nonlinear behavior based on the approach detailed on Figure 1. A combination of virtual modeling and mathematical analysis has been used to identify the linear model of the system. Based on the fundamental hydraulic and dynamic equations of the system, a virtual model has been developed. This model is used to provide a test bench for identifying the order of the system.

Meanwhile, an analytical study of the equations is carried out to define an enhanced input-output relation of the system. Finally, a system identification technique is used to validate the linearized model.

This paper is organized as follows; section 4 briefly describes the architecture of the IEHA electro-hydraulic actuator, IEHA. Section 5 introduces the fundamental hydraulic and mechanical equations used in the development of the virtual model of the

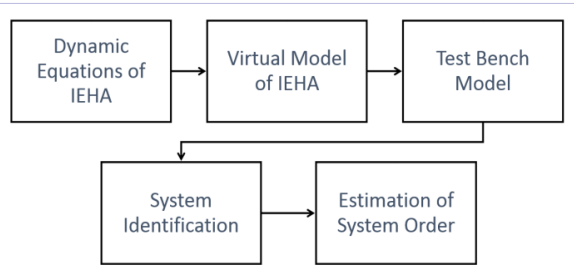

Figure 1: The adopted approach for the IEHA dynamic analysis and identification.

actuator. In Section 6, the virtual model of the system under MATLAB-Simulink is developed and the influence of the internal parameters is studied. A mathematical analysis of the inputoutput relation of the system is presented in Section 7. In Section 8 , a linearized model of the system is achieved through black-box system identification. This linear model is used in Section 9 to implement a predictive position controller for the actuator. The conclusion and the future work are shown in Section 10.

\section{The Architecture of the IEHA}

The integrated hydraulic actuator (IEHA) is based on the power transmission from an electric motor to a hydraulic actuator. The basic idea consists of converting the electric power to mechanical one by using a highly integrated micropump producing pressure and flow (shown in Figure 2). This energy converter contains an in-built micro-valve that controls the eccentricity. The radial pump is connected to the output piston (linear or rotary) through a passive distributor. In order to simplify the figure, only two of the pump's micro-pistons are shown. Since the passive distributor response is very fast compared to the rest of the components, its dynamics can be neglected in the present study. In this section, the functioning principle of the micro-pump and the micro valve are detailed.

\section{The Radial Micro-Pump}

To produce hydraulic energy in the authors proposed solution [1], a micro radial pump is used to deliver the hydraulic power required. The flow discharge of such pump can be controlled by modifying the eccentricity of the main shaft driven by the electric motor with respect to the housing. For a given direction of rotation of shaft, fluid enters the pump, and the centrifugal forces and hydraulic pressure push the micro-pistons to the walls of the housing during half of the rotation cycle, $[0, \pi]$, where the corresponding micro-pistons are connected to the intake port. As the rotor continues around, the vanes sweep the fluid to the opposite side. For the other half of the cycle $[\pi, 2 \pi]$, the micropiston volume decreases and fluid exits the discharge port. Figure 3 presents a simplified IEHA model with two micro-pistons. The distance between the bottom of chamber and the center of the shaft is denoted $\mathrm{d}$, while $\mathrm{Rb}$ is the radius of the carriage. $\mathrm{Hp} 1$ is the distance between the micro-piston of length lp at the dead bottom position and the bottom of its chamber. In the same way, Hp2 is the distance between the micro-piston at the high dead point and the bottom of its chamber as given in Equations 1 and 2 . The piston stroke is defined by the volume of fluid produced during a rotation for a given eccentricity (E). In order to calculate 


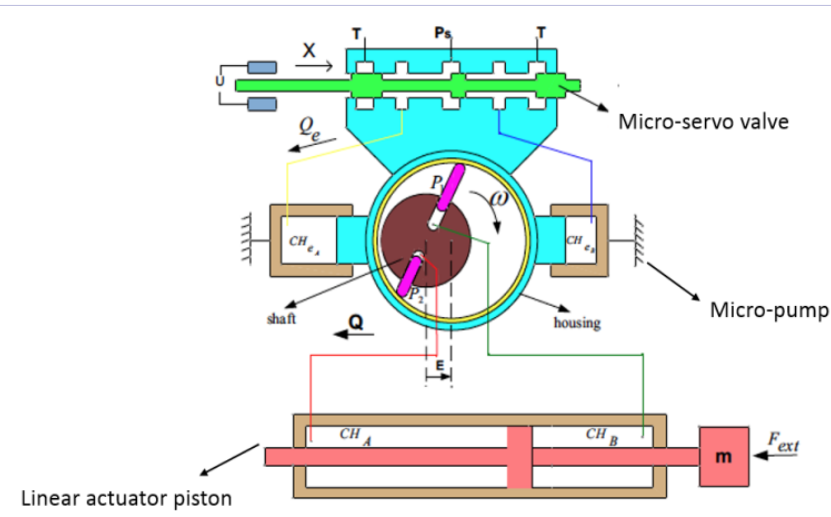

Figure 2: Simplified IEHA diagram with a linear hydraulic cylinder moving a mass subjected to external force $F_{e x t}$.

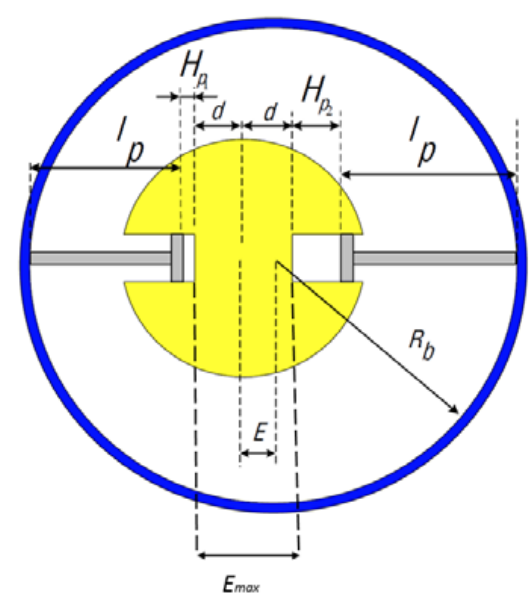

Figure 3: Simplified model of the shaft with two micro-pistons turning inside the housing.

the variation of the micro-pump stroke, the distance that the piston travels during half-rotation corresponding to either the intake or the discharge is determined.

$$
\begin{gathered}
H_{p 1}=R_{b}-E-l_{p}-d \\
H_{p 2}=R_{b}+E-l_{p}-d
\end{gathered}
$$

\section{Micro-valve}

Moving the carriage from one position to another between the two extreme values of the eccentricity, E_max, changes the micropiston stroke and the flow produced by the micro-pump. The micro-valve has the role of adjusting the value of the eccentricity. For the latter, as shown in Figure 2, two simple effect jacks CHeA and $\mathrm{CHeB}$ are integrated on the sides of the carriage to move it with respect to the fixed frame.

By activating the voice-coil of the micro-valve in positive or negative direction, $\mathrm{CHeB}$ is connected to high pressure fluid line $\mathrm{Ps}$, while $\mathrm{CHeA}$ is connected to the return line, and vice versa. In order for the eccentricity to follow the value of input displacement
$\mathrm{X}$, a closed loop system is needed. This closed loop circuit has been carried out mechanically, by connecting the micro-valve external fixed part to the pump housing. Therefore, when $\mathrm{X}$ changes, the micro-valve is opened and $E$ changes until it reaches the value of $\mathrm{X}$, where the micro-valve closes and eccentricity remains constant at this value. An external force applied to the actuator increases the pressure in the output piston, and consequently the micro-pump pistons. This increase in pressure, increases the force applied by the micro-pistons on the housing, and can change the eccentricity. In this case the micro valve opens which will correct the eccentricity and bring it back to the same value as X.

\section{The Dynamic Equations of the IEHA}

In this section, the necessary hydraulic and mechanical equations of the dynamic model are presented. In addition to the leakage, the compressibility parameters and the microscopic flow equations of the micro radial pump are taken into account. These equations are based on the prototype of IEHA where the micro radial pump delivers flow from a high-pressure supply to one side of the cylinder at each instant. The other side of the cylinder is connected to an atmospheric reservoir. The way the micro radial pump is connected to the output cylinder (chamber A or B) depends on the sign of the eccentricity and is done by the passive distributor. The dynamics of the passive distributor are not taken into account, because it has a very fast response time compared to the rest of the system.

\section{Fundamental Hydraulic Equations}

(a) The radial micro-pump

Each micro-piston in the radial pump is connected to the intake line during half of each rotation, where it sucks oil. During the second half, it pushes oil to one of the two cylinder chambers. The micro-pistons are pushed to the carriage wall by centrifugal forces. The microscopic model of flow is:

$$
Q_{p i}=\left\{\begin{array}{cc}
S_{p i} H_{p_{i}} & \theta \dot{o}[0, \pi] \\
-S_{p i} H_{p_{i}} & \theta \text { ò }[\pi, 2 \pi]
\end{array}\right.
$$

$$
Q_{\text {mic }} \quad \sum Q_{p i}
$$

Where Spi is the active area of a single micro-piston, and Hpi is the time derivative of $\mathrm{Hpi}$, the height of oil in micro-piston chamber i at each instant. Hpi is calculated through a geometrical study of the movement of the micro-piston inside the housing. To make the calculation simpler, we define Lpi, as the distance between the shaft center and the micro-piston contact point on the surface of the housing, shown in Figure 4.

$$
L_{p i}=d+H_{p i}+l_{p}
$$

As shown in Figure 4, $\theta$, the angle between the micro-piston and the reference axe, is a function of time and the rotational speed of the shaft $\omega$ and equals $\omega t+\varphi \mathrm{i}$. Using the Pythagorean Theorem, Lpi is calculated as a function of eccentricity: 


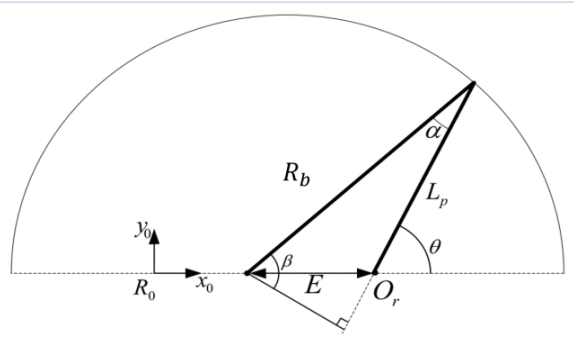

Figure 4: Geometrical study of the moving micro-piston inside the housing.

$$
L_{p i}=\sqrt{R_{b}^{2}-E^{2} \sin ^{2}\left(\omega t+\varphi_{i}\right)}-E \cos \left(\omega t+\varphi_{i}\right)
$$

By taking the time derivative, the speed of micro-piston i is obtained as:

$$
\begin{aligned}
& H_{p_{i}}=L_{p_{i}}=-\dot{E} \cos \left(\omega t+\varphi_{i}\right)+E \omega \sin \left(\omega t+\varphi_{i}\right)- \\
& \frac{E \dot{E} \sin ^{2}\left(\omega t+\varphi_{i}\right)+E^{2} \omega \sin \left(\omega t+\varphi_{i}\right) \cos \left(\omega t+\varphi_{i}\right)}{\sqrt{R_{b}^{2}-E^{2} \sin ^{2}\left(\omega t+\varphi_{i}\right)}}
\end{aligned}
$$

Using the data for the minimum and maximum chamber volume, according to Equations 1 and 2, it can be seen that in each rotation, one single micro-piston delivers an amount of oil equal to:

$$
\left(H_{p 2}-H_{p 1}\right) S_{p i}=2 E S_{p i}
$$

Hence, the average macroscopic flow of $\mathrm{N}$ micro-pistons can be expressed as:

$$
Q_{m a c}=2 N S_{p} E \omega
$$

\section{(b) Micro-valve}

The flow from the micro-valve into the carriage chambers follows the Bernoulli's equation and is a function of the instant opening displacement of the valve $(\mathrm{X}(\mathrm{t})-\mathrm{E}(\mathrm{t}))$, and the actual pressure $\zeta$ in the chamber:

$$
Q_{e}=C_{d} 2 \pi r_{\text {tig }}(X-E) \sqrt{\frac{2}{\rho}} \sqrt{\frac{P_{s}}{2}-\zeta}
$$

Where Cd is the vena contracta coefficient, rtig is the microvalve radius, while $\rho$ is the oil density, and Ps is the high-pressure supply line.

\section{Additional hydraulic parameters}

\section{(a) Compressibility of Hydraulic Oil}

The oil compressibility is defined as the relative change in oil volume per unit change in pressure. Oil compressibility should be taken into account when response time and high-precision control of hydraulic actuators are important. The resistance of a fluid to being compressed is defined by the variable, Bulk Modulus ( $\beta$ ), which is the inverse of compressibility as shown in Equation 10.

$$
\dot{p}=-\beta \frac{\dot{V}}{V}
$$

Where $\mathrm{P}$ and $\mathrm{V}$ represent fluid pressure and volume, respectively. Higher values of $\beta$ make the hydraulic system stiffer, and easier to control. However, in robotic applications, especially in human-robot interaction, it is not desirable to have very stiff actuation, due to safety issues. This leads to increase in response time, and may make the system unstable. These undesirable phenomena can be compromised with a good choice of the other system variables (such as the number of micropistons, or maximum value of eccentricity), combined with an efficient control strategy. Compressibility has a spring-like effect on the movement of the micro-piston, and is taken into account at all levels of the IEHA design.

\section{(b) Hydraulic Oil Leakage}

In contrarily to compressibility, leakage has a damping effect. In carriage and output cylinder, this leakage is from the highpressure chamber to the low pressure one. In the micro-pistons, the leakage takes place from the hydraulic chamber to the micro pump body. In the latter, this leakage inhibits a large increase in fluid pressure, when connected to the intake channel. The leakage flow QleakA is given by the following relation:

$$
Q_{\text {leakA }}=K_{\text {in }}\left(P_{A}-P_{B}\right)+K_{e x}\left(P_{A}\right)
$$

Where Kin and Kex are the internal and external leakage

Coefficients respectively. These two values are a function of the surface of the leakage section.

\section{Motion Dynamics}

\section{(a) Carriage Eccentricity}

The dynamic equation of the carriage, using eccentricity $\mathrm{E}$ can be written as:

$$
F_{e}+F_{p}=m_{e} \ddot{E}
$$

Where Fp is the force exerted by the oil pressure in the radial pump on the carriage, while $\mathrm{Fe}=2 \mathrm{Se} \zeta$ is the hydraulic force in the carriage chambers. $2 \zeta$ is the pressure difference between its two sides, which changes as a function of the compressibility and the real flow Qe in the carriage which is given by:

$$
Q_{e}=S_{e} \dot{E}+\frac{v_{e}}{\beta} \dot{\zeta}-Q_{\text {eleak }}
$$

Where Qeleak is the leakage from the carriage micro-pistons to the body of the actuator. ve and $\beta$ are the chamber volume and the bulk modulus of the oil respectively.

\section{(b) Fluid pressure in the radial micro-pump}

For a given eccentricity, one side of the pump is directly connected to the supply terminal from the micro-valve, and the 
other side of the micro-pump is connected to one chamber of the output actuator. Each micro-piston is connected to the supply line in the first half of its rotation, where it aspires fluid. In the second half, it ejects the fluid to the cylinder chamber. However, in the presence of piston leakages, Qpileak, the pressure increase in the micro-pistons is not very large. For each micro-piston $\mathrm{Pi}$ we can consider:

$$
C_{d} \Pi r_{r p}^{2} \sqrt{\frac{2}{\rho}} \sqrt{\Delta P_{i}}=Q_{p i}+\frac{v_{p i}}{\beta}-Q_{p i_{\text {eak }}}
$$

With,

$$
\Delta p_{i}=\left\{\begin{array}{ccc}
P_{r p}-P_{p i} & \theta & \text { ò }[0, \pi] \\
P_{p i}-P_{c \lambda} & \theta & \grave{o}[\pi, 2 \pi]
\end{array}\right.
$$

where rrp is the radius of the in-out opening section of the micro pump intake tube, vpi is the volume of Pi, Prp is the pressure of the intake channel of the pump, and $\lambda \in\{A, B\}$ represents chamber $A$ or $B$ of the output cylinder, and depends on the sign of the eccentricity. In addition to $\beta, \mathrm{Qpi}, \mathrm{Cd}, \rho$ and Qpileak which are bulk modulus of elasticity, flow inside micropiston, venta contracta constant, density of the oil and internal leakage of the micro-piston respectively. The fluid pressure in micro-pistons exerts a force on the carriage that can be expressed as:

$$
F_{p}=\left(P_{r p}-P_{c}\right) S_{p} \cos (\omega t)
$$

\section{(c) Position of the linear actuator}

The dynamic equation of the hydraulic linear actuator is given by Equation 17, where F_c $=2$ S_c P_c and F_ext are the hydraulic force and the external force exerted on the cylinder respectively. $\mathrm{M}$ and $\mathrm{Y}$ are the end-effector mass and position respectively. 2Pc is the pressure difference between the two cylinder chambers $\mathrm{A}$ and $\mathrm{B}$ and Sc is the surface area of the linear hydraulic piston cylinders.

$$
F_{c}-F_{\text {ext }}=m \ddot{Y}
$$

Pressure change due to compressibility and the input flow is defined as:

$$
Q=S_{c} \dot{Y}+\frac{v_{c}}{\beta} P_{c}-Q_{\text {leak }}
$$

Where $Q$ is the flow from the micro-pump. Qleak is the internal leakage between the two chambers of the cylinder. vc is the volume of the chamber of the output cylinder.

\section{IEHA Virtual Model}

Compactness of IEHA makes it difficult to measure the internal variables of the system, such as the micro pump eccentricity and internal pressure drop. Moreover, it is important to study the effect of configuring the internal variables (e.g. the dimensions of different mechanical parts) on the behavior of the system before modifying the system design in future prototypes. Therefore, development of a virtual model of the IEHA provides deeper understanding of the system and can be considered as an efficient platform for further system optimization. The effectiveness of the developed virtual model allows a quick and accurate evaluation of the internal parameters early in the design and development stage.

For this purpose, MATLAB-Simulink was used to model all the dynamic equations of the IEHA actuator, as well as fluid properties such as bulk modulus and internal leakage as shown in Appendix I. The blocks on the left-hand side of the figure represent the dynamics of the micro-valve, which takes signal $\mathrm{X}$ as an input, the resulting eccentricity $\mathrm{E}$ is applied to the micropump which is simulated as 15 individual micro-pistons (middle blocks). The flow produced by the micro-pistons is sent to the passive distributor which directs it to side A or B of the linear actuator (right hand side blocks). The electric motor has been modeled as constant input rotating at a fixed speed. The used parameters values are given in Table 1 .

The following simulation results show the variation of internal parameters against a step input of $0.04[\mathrm{~cm}]$ of the micro valve position X. Generally, the input command X decides how much the micro-valve should be opened and as the pressure in the chamber increases, the carriage moves and E changes. Figure 5 shows the response of the carriage eccentricity, E, while it follows the micro-valve displacement X. As seen in Figure 5, the time response of $\mathrm{E}$ is around 0.007 [s] and it follows the input $\mathrm{X}$ with a negligible delay.

In order to illustrate the pressure variation in the micropistons, the variations of the output cylinder pressures are linked to the oscillation of flow. By looking at pump flow curve (Figure 6 ), it is clear that the oscillates with a frequency of $10[\mathrm{~Hz}]$. This oscillation is due to the fluid rippling from the micro-radial pump. There is also a slight peak in the beginning, the faster the eccentricity the bigger this peak of flow is.

In the beginning of the cylinder's movement as shown in Figure 7, the pressure across the cylinder increases due to fluid compressibility. And as the load starts to move, the pressure difference across the cylinder drops and the velocity reaches the stable value of $2.2[\mathrm{~cm} / \mathrm{s}]$ as shown Figure 8 . The pressure difference across the output stays positive ( $0.8 \mathrm{bar})$. This is

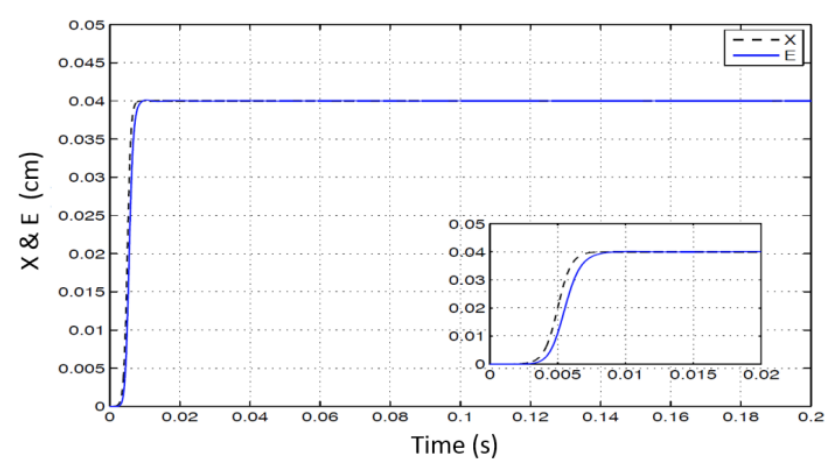

Figure 5: Input $\mathrm{X}$ to the micro-valve and the carriage eccentricity $E$ following $X$. The response time of $E$ is 0.007 [s]. 
Table 1: The parameter values used in the virtual model of IEHA.

\begin{tabular}{|c|c|c|}
\hline PARAMETER & Physical Quantity & Value \\
\hline$P_{s}$ & Supply pressure of micro-valve & $10 \mathrm{bar}$ \\
\hline$r_{\text {tig }}$ & Micro-valve radius & $0.25 \mathrm{~cm}$ \\
\hline$m_{e}$ & Carriage mass & $0.091 \mathrm{~kg}$ \\
\hline$S_{e}$ & Carriage active surface area & $1.644 \mathrm{~cm}^{2}$ \\
\hline$E_{\max }$ & Maximum eccentricity & $0.05 \mathrm{~cm}$ \\
\hline$v_{e}$ & Chamber volume of carriage & $0.0822 \mathrm{~cm}^{3}$ \\
\hline$\beta$ & Bulk modulus & $800 \mathrm{MPa}$ \\
\hline$C_{d}$ & Vena contracta coefficient & 0.62 \\
\hline$\rho$ & Fluid density & $840 \mathrm{~kg} / \mathrm{m}^{3}$ \\
\hline$\omega$ & Electric motor rotational speed & $3000 \mathrm{rpm}$ \\
\hline$P_{r p}$ & Supply pressure of micro-pump & $10 \mathrm{bar}$ \\
\hline$N$ & Number of micro-pistons & 15 \\
\hline$r_{r p}$ & Micro-pump in-out radius & $0.2 \mathrm{~cm}$ \\
\hline$m_{p}$ & Micro-piston mass & $0.45 \mathrm{~g}$ \\
\hline$S p$ & Micro-piston surface area & $0.197 \mathrm{~cm}$ \\
\hline$L_{p}$ & Micro-piston stroke & $0.27 \mathrm{~cm}$ \\
\hline$R_{b}$ & Interior ring radius & $0.3 \mathrm{~cm}$ \\
\hline$d$ & Distance between chamber & $0.4 \mathrm{~cm}$ \\
\hline$m$ & bottom \& shaft center & $10 \mathrm{~kg}$ \\
\hline$v_{c}$ & Load mass & $23.562 \mathrm{~cm}$ \\
\hline$S_{c}$ & Chamber volume of output & cylinder \\
\hline
\end{tabular}

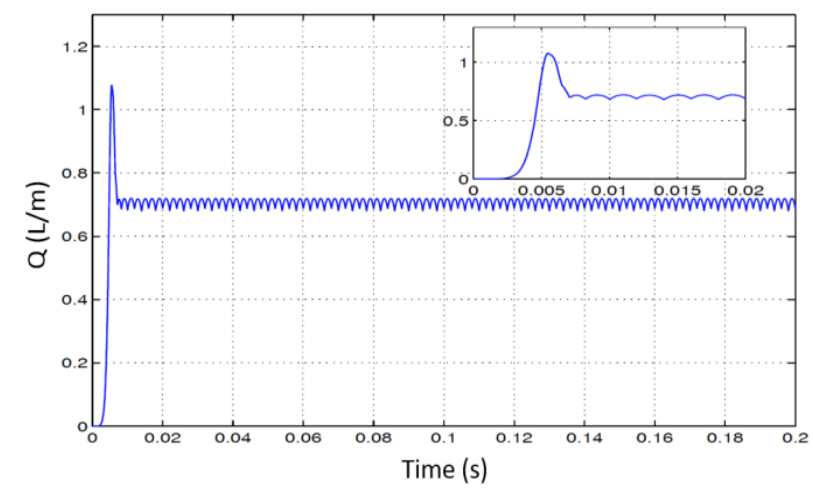

Figure 6: Flow produced by the micro-radial pump.

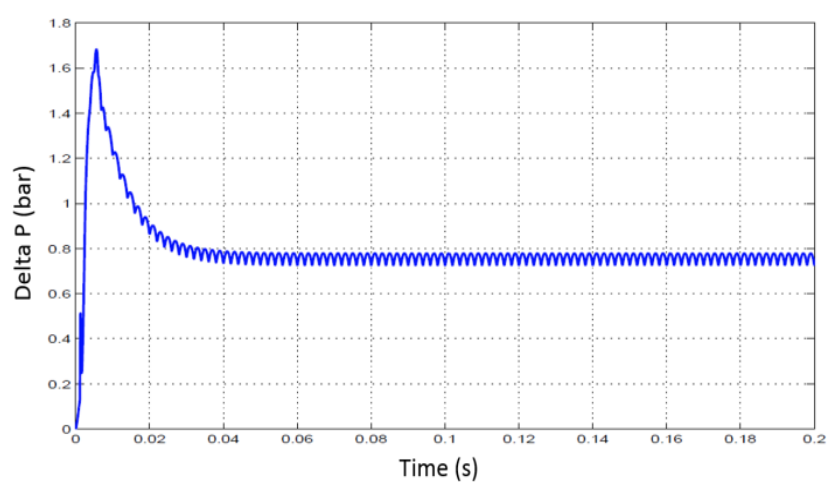

Figure 7: Pressure difference in the chambers of the output cylinder, $P_{c y l A}-P_{c y l B .}$

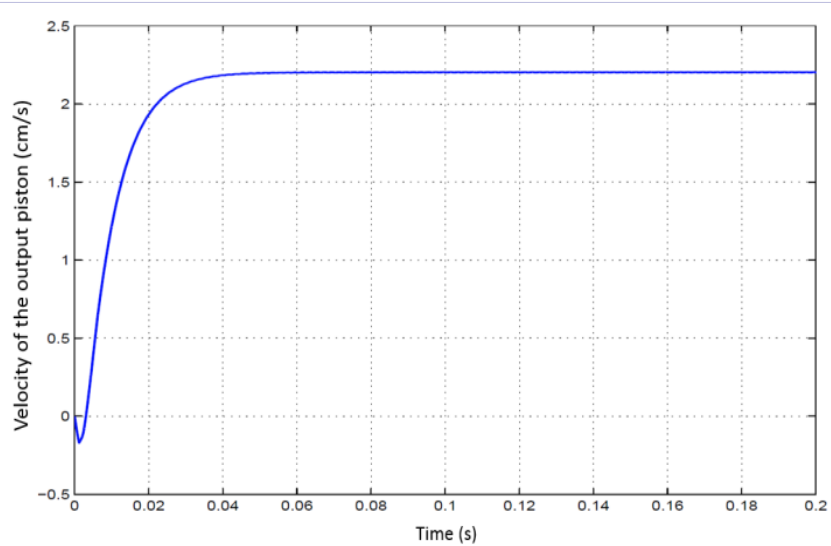

Figure 8: Velocity of the output piston $Y$.

because of the asymmetry of the output piston ScylA which is almost half ScylB. This will lead also to a gradual change in the output piston position $\mathrm{Y}$ as shown in Figure 9. This is continued till the occurrence of a change in input of the micro-valve signal.

In these results the output flow of the pump seems to immediately increase the pressure force in the output cylinder and make the piston velocity increase to a stable value. This is because the dynamics of the passive distributor are not taken into account in this model in Simulink.

\section{The input/output model}

\section{Dynamic Equations}

The ultimate goal of this section is to estimate the model order of the IEHA. As will be seen in subsection 8, this order combined with the test bench, are used to find a linearized function of the proposed IEHA, through system identification methods. Since our goal is to find the simplest low order form of equations equivalent to the system, internal leakage has been neglected in the following analysis. This is due to the compactness of the system.

Returning to the equations in subsection 5-5.3, if Pc is derived and replaced from Equation 17 into Equation 18, and by considering the macroscopic value of flow according to Equation 8, we will have the eccentricity E expression in terms of Fext, Y, and their derivatives:

$$
E=\frac{S_{c}}{2 N S_{p} \omega} \dot{Y}+\frac{1}{2 N S_{p} \omega} \frac{v_{c}}{\beta} \frac{1}{2 S_{c}} m Y^{(3)}+\frac{1}{2 N S_{p} \omega} \frac{v_{c}}{\beta} \frac{1}{2 S_{c}} \dot{F}_{\text {ext }}
$$

Where Y3 and Y5 are the payload jerk and the second derivative of the jerk respectively.

On the other hand, the force of the micro-pump pistons exerted on the carriage chambers is given by Equation 16. By replacing Pc, the micro pump's force can also be written in terms of the output position, the external force, and their derivatives:

$$
F_{p}=\left(P_{r p}-\frac{1}{2 S_{c}}\left(m \ddot{Y}+F_{e x t}\right)\right) S_{p} \cos (w t)
$$




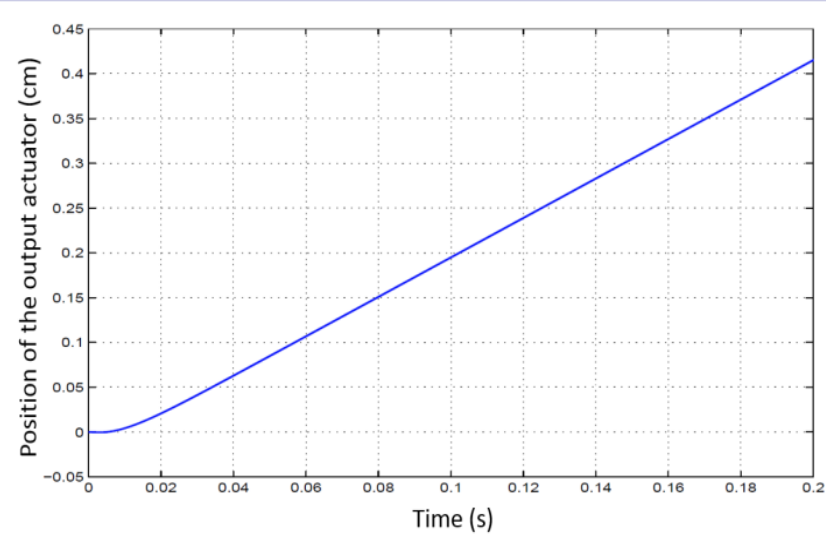

Figure 9: Position of the output piston $Y$.

The pressure difference in carriage chambers can be written in terms of Ë and Fp according to Equation 12:"

$$
\zeta=\frac{1}{2 S_{e}}\left(m_{e} \ddot{E}-F_{p}\right)
$$

Deriving Ë from Equation $19, \zeta$ can also be written in terms of Y and Fext :

$$
\begin{aligned}
& \zeta=\frac{1}{2 S_{e}}\left(\frac{m_{e} S_{c}}{2 N S_{p} \omega} Y^{(3)}+\frac{m_{e}}{2 N S_{p} \omega} \frac{v_{c}}{\beta} \frac{1}{2 S_{c}} m Y^{(5)}+\frac{m_{e}}{2 N S_{p} \omega} \frac{v_{c}}{\beta} \frac{1}{2 S_{c}} F_{e x t}^{(3)}-\right. \\
& \left.\left(P_{r p}-\frac{1}{2 S_{c}}\left(m \ddot{Y}+F_{e x t}\right)\right) S_{p} \cos (\omega t)\right)
\end{aligned}
$$

Fext(3) is the third-time derivative of the external force while $Y(3)$ and $Y(5)$ are the payload jerk and the second derivative of the jerk. By replacing the Bernoulli equation of flow of the microvalve (Equation 9) in the dynamic equation (Equation 12), we can write an expression which relates the input variable $\mathrm{X}$, to the internal variables $\mathrm{E}$ and $\zeta$ :

$$
2 \Pi C_{d} r_{\text {tig }} \sqrt{\frac{2}{\rho}}(X-E) \sqrt{\frac{P_{s}}{2}-\zeta}=S_{e} \dot{E}+\frac{v_{e}}{\beta} \dot{\zeta}
$$

Replacing E and $\zeta$ from Equation 19 and Equation 22 in the latter, we will obtain a non-linear equation that represents the relationship between the system input, micro-valve position $\mathrm{X}$, and the measurable values at the end-effector, $Y$, Fext, and their derivatives.

$$
X=A+\frac{B}{C}
$$

Where,

$$
\begin{aligned}
& A=K_{10} \dot{Y}+K_{11 m} Y^{(3)}+K_{11} F_{e x t} \\
& B=K_{8}\left(K_{10} \ddot{Y}+K_{11 m} Y^{(4)}+K_{11} \ddot{F_{e x t}}\right)+ \\
& K_{9}\left(K_{13} Y^{(4)}+K_{14 m} Y^{(6)}+K_{14} F_{e x t}^{(4)}\right)-\left(K_{16}+K_{17 m} \ddot{Y}+K_{17} F_{e x t}\right) \\
& \sin (\omega t)+K_{18 m} \cos (\omega t) Y^{(3)}+K_{18} \cos (\omega t) \dot{F}_{e x t}
\end{aligned}
$$

$$
C=K_{7} \sqrt{\begin{array}{c}
1+K_{1} Y^{(3)}+K_{2 m} Y^{(5)}+K_{2} F_{e x t}^{(3)} \\
+K_{3} \cos (\omega t)+K_{4 m} \ddot{Y} \cos (\omega t)+K_{4} F_{e x t} \cos (\omega t)
\end{array}}
$$

The coefficients $\mathrm{K} 1$ to $\mathrm{K} 20$ are all constants and depend on the dimensions of the actuator and the characteristics of the fluid. These coefficients are given as follows:

$$
\begin{gathered}
K_{1}=\frac{m_{e} S_{c}}{4 P_{s} S_{e} S_{p} N \omega} \quad K_{2 m}=\frac{m_{e} v_{c} m}{8 P_{s} S_{e} \beta S_{c} S_{p} N \omega} \\
K_{2}=\frac{m_{e} S_{c}}{8 P_{s} S_{e} \beta S_{c} S_{p} N \omega} \\
K_{3}=\frac{S_{p}}{2 S_{e}} \quad K_{4 m}=-\frac{S_{p} m}{4 S_{e} S_{c} P_{s}} \quad K_{4}=-\frac{S_{p}}{4 S_{e} S_{c} P_{s}} \\
K_{7}=2 \Pi C_{d} r_{\text {tig }} \sqrt{\frac{P_{s}}{\tilde{n}}} \beta \quad K_{8}=S_{e} \beta \quad K_{9}=\frac{v_{e}}{2 S_{e}}
\end{gathered}
$$

$$
\begin{gathered}
K_{10}=\frac{S_{c}}{2 S_{p} N \omega} \quad K_{11 m}=\frac{v_{c} m}{4 \beta S_{c} S_{p} N \omega} \\
K_{11}=\frac{v_{c}}{4 \beta S_{c} S_{p} N \omega}
\end{gathered}
$$

$$
\begin{gathered}
K_{13}=\frac{S_{c} m_{e}}{2 S_{p} N \omega} \quad K_{14}=\frac{v_{c} m_{e}}{4 \beta S_{c} S_{p} N \omega} \\
K_{14}=\frac{v_{c} m_{e} m}{4 \beta S_{c} S_{p} N \omega}
\end{gathered}
$$

$$
\begin{gathered}
K_{15}=\frac{v_{c} m_{e}}{4 \beta S_{c} S_{p} N \omega} \quad K_{16}=-P_{r p} S_{p} \omega \\
K_{17 m}=\frac{S_{p} \omega m}{2 S_{c}}
\end{gathered}
$$

$$
K_{17}=\frac{S_{p} \omega}{2 S_{c}} \quad K_{18 m}=\frac{S_{p} m}{2 S_{c}} \quad K_{18}=\frac{S_{p}}{2 S_{c}}
$$

\section{Model Reduction}

By combining the coefficients in Equation (24), the system is found to be non-linear and of order six. However, with an analytical study of the coefficients of the high order derivatives. Some of these terms are of very low orders of amplitude compared to the others. These terms can be neglected without having a major effect on the behavior of the actuator performance. 


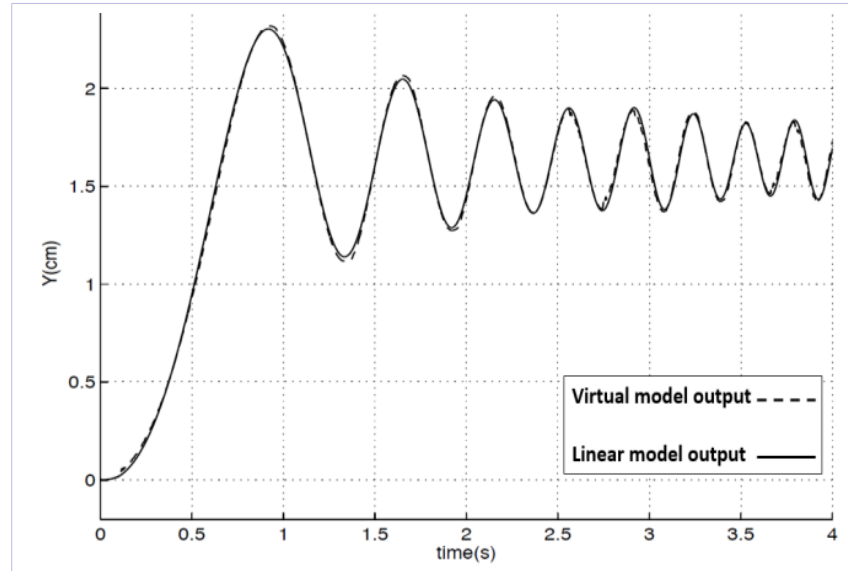

Figure 10: Comparison of the outputs from the linear model of equation (26) and the virtual model for chirp signal input.

By comparing the coefficients $\mathrm{K}(1 \ldots 4)$, we see that the terms $\mathrm{K} 2$ and $\mathrm{K} 2 \mathrm{~m}$ will always be very small compared to the others, because they are divided by the bulk modulus, which is of an order between 107 and 109, depending on the type of the used oil. Therefore, these two terms will always be very small. With the same analysis for the terms in B, K14 and K14m are always very small, regardless of the actual dimensions of the actuator. Therefore, these terms have a small influence on the dynamics of the system and can be neglected. The resulting input-output equation is found to be of a fourth order and can be presented as:

$$
X=A+\frac{B_{s}}{C_{s}}
$$

Where the new expression of Bs and Cs are the following (Coefficient A remains the same):

$$
\begin{aligned}
& B_{s}=K_{8}\left(K_{10} \ddot{Y}+K_{11 m} Y^{(4)}+K_{11} F_{\text {ext }}\right)+K_{9}\left(K_{13} Y^{(4)}\right)- \\
& \left(K_{16}+K_{17 m} \ddot{Y}+K_{17} F_{\text {ext }}\right) \sin (\omega t)+K_{18 m} \cos (\omega t) Y^{(3)}+ \\
& K_{18} \cos (\omega t) F_{\text {ext }} \\
& C_{s}=K_{7} \sqrt{\begin{array}{c}
1+K_{1} Y^{(3)}+K_{3} \cos (\omega t)+ \\
K_{4 m} \ddot{Y} \cos (\omega t)+K_{4} F_{e x t} \cos (\omega t)
\end{array}}
\end{aligned}
$$

\section{Identification and Analysis of the Linear System}

Hydraulic actuators are generally complex and have nonlinear dynamics, which makes the design of feedback controllers more difficult. Therefore, our approach is to use the input-output data sets, obtained from the numerical simulation of the virtual system, presented in section 6 , combined with the system order found analytically in section 7 , to estimate a linear model of the proposed IEHA.

\section{Preparation of the Test-Bench}

The estimation data sets are obtained from the virtual model of the actuator, described in section 6. We have a test bench of double input single-output data. The inputs signals to this test bench include a single pulse, a step, and chirp signal from 0.1 $10 \mathrm{~Hz}$ as inputs for $\mathrm{X}$. In all the simulated runs, temperature and the compressibility of the fluid are assumed to be constant.

\section{Identification of the Linear System}

MATLAB system identification toolbox has been used to estimate a linear transfer function that best describes the behavior of the system. This toolbox estimates model parameters using iterative prediction-error minimization method (PEM). PEM is an iterative estimation command, using non-linear least squares algorithm to minimize the cost function.

The system input-output linear function has been identified with variable load (can also be interpreted as being in contact with the environment). Unlike simple micro-valves, dynamics of IEHA depends on the load (this is also the property that makes it back-drivable). Increase in the external force, causes increase of the cylinder pressure, which will increase the pressure in the micro-pistons. Consequently, the oil pressure on the carriage goes up and this can displace the carriage, (i.e. modify the eccentricity E). Hence, the output flow of the micro-pump is changed. This effect is also seen in the analytical relation between $\mathrm{Y}$ and $\mathrm{X}$. Therefore, we consider Fext as an input to our system. Different external forces were applied to the output stroke. These forces included a step and a chirp signal of maximum frequency of $10 \mathrm{~Hz}$, with amplitude of $100 \sim 1000 \mathrm{~N}$. Inserting $\mathrm{X}$ and Fext as two separate inputs to the system, a new linear model has been estimated as follows:

$$
\begin{aligned}
& Y(s)=G_{1}(s) X+G_{2}(s) F_{\text {ext }} \\
& G_{1}(s)=\frac{K}{s\left(1+\lambda_{1} s\right)\left(1+\lambda_{2} s\right)\left(1+\lambda_{3} s\right)} \\
& G_{2}(s)=\frac{K^{\prime}}{s\left(1+\lambda_{1}^{\prime} s\right)}
\end{aligned}
$$

Where

$$
\begin{aligned}
& \lambda=10^{-3}[1.14,1.14,1.11] \\
& \lambda_{1}^{\prime}=0.43 \times 10^{-6} \\
& \mathrm{~K}=115.4 \\
& \mathrm{~K}^{\prime}=6.3 \times 10^{-4}
\end{aligned}
$$

The system found here is of fourth order, which corresponds to our findings in the previous section. To illustrate the accuracy of the model above, the inputs and the output of the virtual model and the linear model, for chirp signal inputs are shown in Figure 11. The output response of the linear system $Y$ is found to be the same as of the virtual model.

To validate the piston response, another simulation is conducted. A step input of $0.04[\mathrm{~cm}]$ is given to the micro-valve, then after $0.5[\mathrm{~s}]$, the micro-valve is closed and the output is suspended at a certain position. Then a varying sinusoidal force is applied on the cylinder, which will pull and push the piston. The results are shown in Figure 13. 


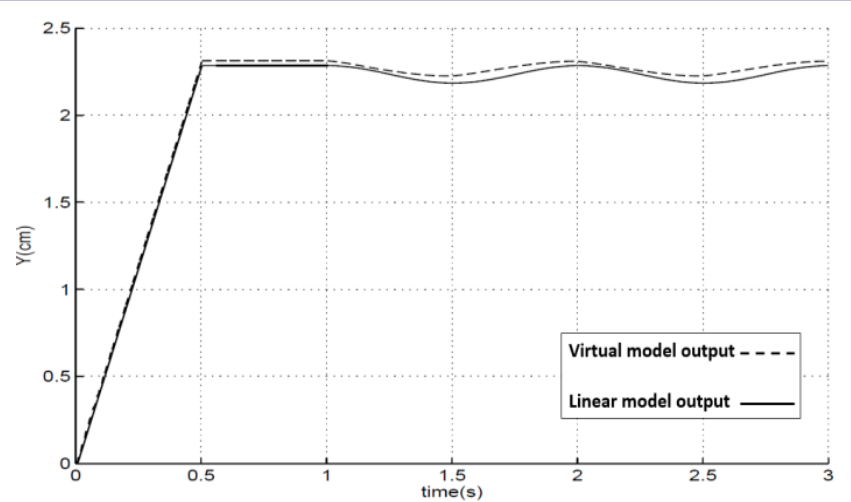

Figure 11: Comparison of the outputs from the linear model of equation (26) and the virtual model, for step input.

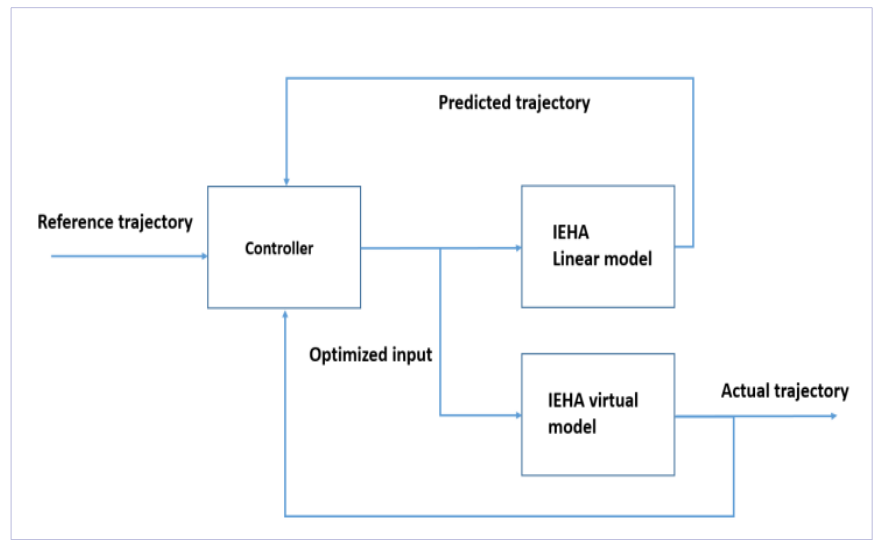

Figure 12: Predictive control block diagram.

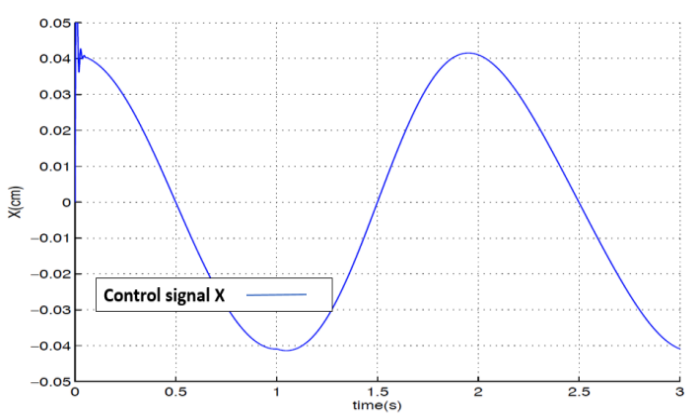

(a)

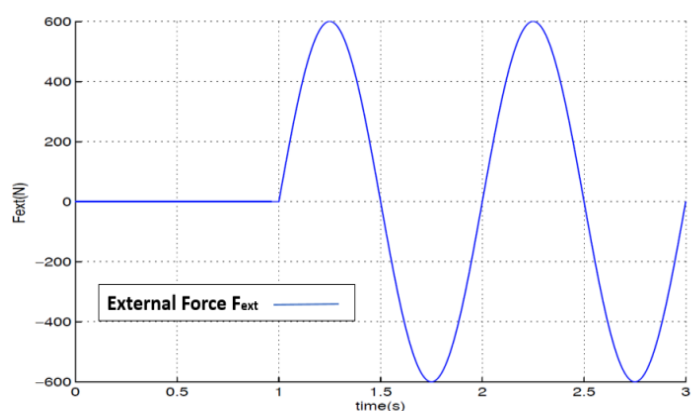

(b)

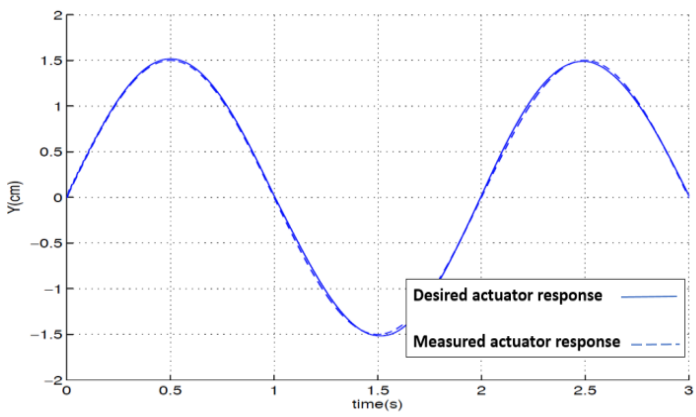

(c)

Figure 13: (a) sinusoid X input, (b) external force $F_{\text {ext }}$ input, (c) Simulated position tracking of loaded actuator.

From those two previous simulation results, the virtual model was compared with the linear model while responding to step, chimp and sinusoidal inputs and proved its worthiness. This validates the response of the reduced order model when compared with the full order one.

\section{Predictive controller implementation}

Many control schemes have been proposed for the control of hydraulic actuators. Simple PID controllers have proven to be inadequate, because of complex non-linarite of hydraulics. Specifically, in the proposed IEHA, the controller signal is very sensitive to the dynamics of the internal parameters, and this makes the PID tuning complicated. Hence, another control method is sought after to control the position of the load actuator.

Since, we have already a linear based model for the IEHA in addition to the virtual model; a model predictive control algorithm 
seems adequate. The model predictive control is a strategy that is based on the explicit use of some kind of linear system model to predict the controlled variables over a certain time period [30] In our case the linear model of the IEHA can be the predicting model. This way we avoid the undesirable dynamics of the actuator in the feedback. Another advantage of this method is that this feedback is faster than the one coming from the nonlinear model. Therefore, the control signal is adjusted by "predicting" the future error. The control block diagram is shown in Figure 12.

\section{Simulation Results}

In the simulation results shown in Figure 13, the position trajectory is a sine wave with frequency $\pi[\mathrm{rad} / \mathrm{s}]$. a sinusoidal force of $600[\mathrm{~N}]$ is applied on the piston. As we can see in Figure $11 \mathrm{c}$, the control signal is modified to compensate for this change in the load and keep the actuator in the commanded position. The resulting response has almost no delay time, negligible overshoot, and insignificant steady state error.

\section{Conclusion}

In this paper a detailed mathematical model and a virtual model for the IEHA have been developed. The system order is obtained and simplified by neglecting the ineffective parameters. An analytical study of IEHA combined with black-boxidentification has been used to find the linear model of this highly complex system. This linear model is used in implementing a predictive controller to show its precision in position tracking. Since the IEHA main application is related human-robot interaction, the external force exerted by the environment is taken as an input of the system.

For future work, the internal parameters of the hydraulic mathematical model such as leakage and compressibility are not constant and vary with time and temperature. Hence, a further work must consider a better solution to identify the internal states of the system. A better use of an adaptive controller accordingly can be pursued. On the other hand, the passive distributor model has to be included in order to get a complete description of the IEHA model. The new virtual model presented can be used for manufacturing other actuator prototypes with all of its inner parameters identified. Finally, the hardware use of the IEHA to actuate an active compliant hydraulic arm is under development. This will enable us to have an integrated hydraulic actuator for the underdevelopment humanoid robot HYDROÏD.

\section{Acknowledgement}

This work was mainly funded by BIA- TURNKEY TEST SYSTEMS Company

\section{References}

1. Alfayad S, Benouedou F, Namoun F. Convertor for converting mechanical energy into hydraulic energy and robot implementing said convertor. US Patent WO/2009/118366. 2009.

2. Semini C, Tsagarakis NG, Guglielmino E, Focchi M, Cannella F, Caldwel DG. Design of HyQ - a hydraulically and electrically actuated quadruped robot. Proc. Inst Mech Eng Part I, J Syst Control Eng. 2011;225:831849. doi: $10.1177 / 0959651811402275$
3. Sakagami Y, Watanabe R, Aoyama C, Matsunaga S, Higaki N, Fujimura K. The intelligent ASIMO: system overview and integration. In Proc. of IROS. 2002:2478-2483. doi: 10.1109/IRDS.2002.1041641.

4. Konno A, Sellaouti R, BEN Amar F, Ouezdou FB. Design and Development of the Biped prototype ROBIAN. ICRA. 2002:1384 1389.

5. Kaneko K, Kanehiro F, Morisawa M, Akachi K, Miyamori G, Hayashi A, et al. Humanoid robot HRP-4 - Humanoid robotics platform with lightweight and slim body. IEEE International Conference for Intelligent Robotic Systems. 2011:4400-4407.

6. Buschmann T, Lohmeier S, Ulbrich H. Humanoid robot Lola: Design and walking control. J Physiol-Paris. 2009;103(3-5):141-148.

7. Tellez R, Ferro F, Garcia S, Gomez E, Jorge E, Mora D, et al. Reem-B : an autonomous lightweight human-size humanoid robot. 8th IEEE International Conference on Humanoid Robots (Humanoids08). 2008. doi: 10.1109/ICHR.2008.4755995

8. Zhou X, Bi S. A survey of bio-inspired compliant legged robot designs. Bioinspir Biomim. 2012;7(4):041001. doi: 10.1088/17483182/7/4/041001.

9. Hurst JW. The role and implementation of compliance in legged locomotion. Dissertation. Carnegie Mellon University. 2008.

10. Pratt JE. Exploiting inherent robustness and natural dynamics in the control of bipedal walking robots. Dissertation, Massachusetts Institute of Technology. 2000.

11. Brooks RA, Breazeal C, Marjanovic M, Scassellati B, Williamson MM. The Cog Project: Building a Humanoid Robot Lect Notes Comput Sci. 1999;1562:52-87.

12. Robinson DW, Pratt JE, Paluska DJ, Pratt GA. Series elastic actuator development for a biomimetic walking robot. Advanced Intelligent Mechatronics. Proceedings. IEEE/ASME International Conference.1999. 561-568.

13. Edsinger-Gonzales A, Weber J. Domo: A Force Sensing Humanoid Robot for Manipulation Research. IEEE International Conference on Humanoid Robots. 2004

14. Albers A, Brudniok S, Ottnad J, Sauter C, Sedchaicharn K. ARMAR III design of the upper body. Technical report. Institute of Product Development University of Karlsruhe (TH). 2006.

15. Raibert M, Blankespoor K, Nelson G, Playter R. Bigdog, the RoughTerrain Quadruped Robot. In Proceedings of the 17th World Congress. 2008;41(2):10822-10825.

16. Bentivegna DC, Atkeson CG, Kim JY. Compliant control of a hydraulic humanoid joint. Proc. 2007 7th IEEE-RAS Int Conf Humanoid Robot. HUMANOIDS 2007. 2008:483-489.

17. Semini C. HyQ - Design and Development of a Hydraulically Actuated Quadruped Robot. Darwin. 2010.

18. Hyon SH, Suewaka D, Torii Y, Oku N, Ishida H. Development of a fast torque-controlled hydraulic humanoid robot that can balance compliantly. Humanoid Robots (Humanoids). 2015 IEEE-RAS 15th International Conference on, 2015:576-581.doi: 10.1109/ HUMANOIDS.2015.7363420.

19. Alfayad S, Ouezdou FB, Namoun F, Cheng G. Highly Integrated ElectroHydraulic Actuator for Robotics - Part II: Theoretical modelling, Simulation, Control \& Comparison with real measurements. Sensors and Actuators A: Physical. 2011;169(1):124-132 
20. Bobrow J E, Desai J. Modeling and Analysis of a High-Torque, Hydrostatic Actuator for Robotic Applications. Experimental Robotics I. 1990:215-228.

21. Habibi S, Goldenberg A. Design of a New High Performance Electrohydraulic Actuator. Proc 1999 IEEE/ASME. 1999;5:158-164.

22. Gnesi E, Maré JC, Bordet JL. Modeling of EHA Module Equipped with Fixed-Displacement Vane Pump. 13th Scand Int Conf Fluid Power. SICFP. 2013:141-153.

23. N. Takahashi, T. Kondo, and M. Takada, Masutani K, Okano S, Tsujita M. Development of prototype electro-hydrostatic actuator for landing gear extension and retraction system. Sumitomo Precision Products Co Ltd. 2008 :165-168.

24. Altare G, Vacca A, Richter C. A Novel Pump Design for an Efficient and Compact Electro-Hydraulic Actuator. In IEEE Aerospace Conference. 2014.

25. Altare G, Vacca A. A Design Solution for Efficient and Compact Electrohydraulic Actuators. Procedia Engineering. 2015;106:8-16.

26. Alfayad S, Ouezdou FB, Namoun F, Cheng G. Lightweight High Performance Integrated Actuator for Humanoid Robotic Applications:
Modeling, Design \& Realization. In Proc IEEE Int Conf on Robotics and Automation (ICRA). 2009.

27. Kazerooni H. Dynamics and control of instrumented harmonic drives. J Dyn Syst Meas Control. 1995;117:15-19.

28. Kaminaga H, Yamamoto T, Ono J, Nakamura Y. Backdrivable miniature hydrostatic transmission for actuation of anthropomorphic robot hands. Humanoid Robots. 2007 7th IEEE-RAS International Conference on. 2007:36-41.

29.Zhu Q, Mao Y, Xiong R, Wu J. Adaptive Torque and Position Control for a Legged Robot Based on a Series Elastic Actuator. Int Journal of Advanced Robotics Systems. 2016:1.

30. Chalupa P, Novák J. Modeling and model predictive control of a nonlinear hydraulic system. Computers \& Mathematics with Applications Journal. 2013;66(2):155-164.

31. Alfayad S, Ouezdou FB, Namoun F, Cheng G. New Highly Integrated Electro-Hydraulic Actuator for Robotics - Part I : Principle, Prototype Design \& First Experiments. Sensors and Actuators A: Physical. 2011;169(1):115-123.

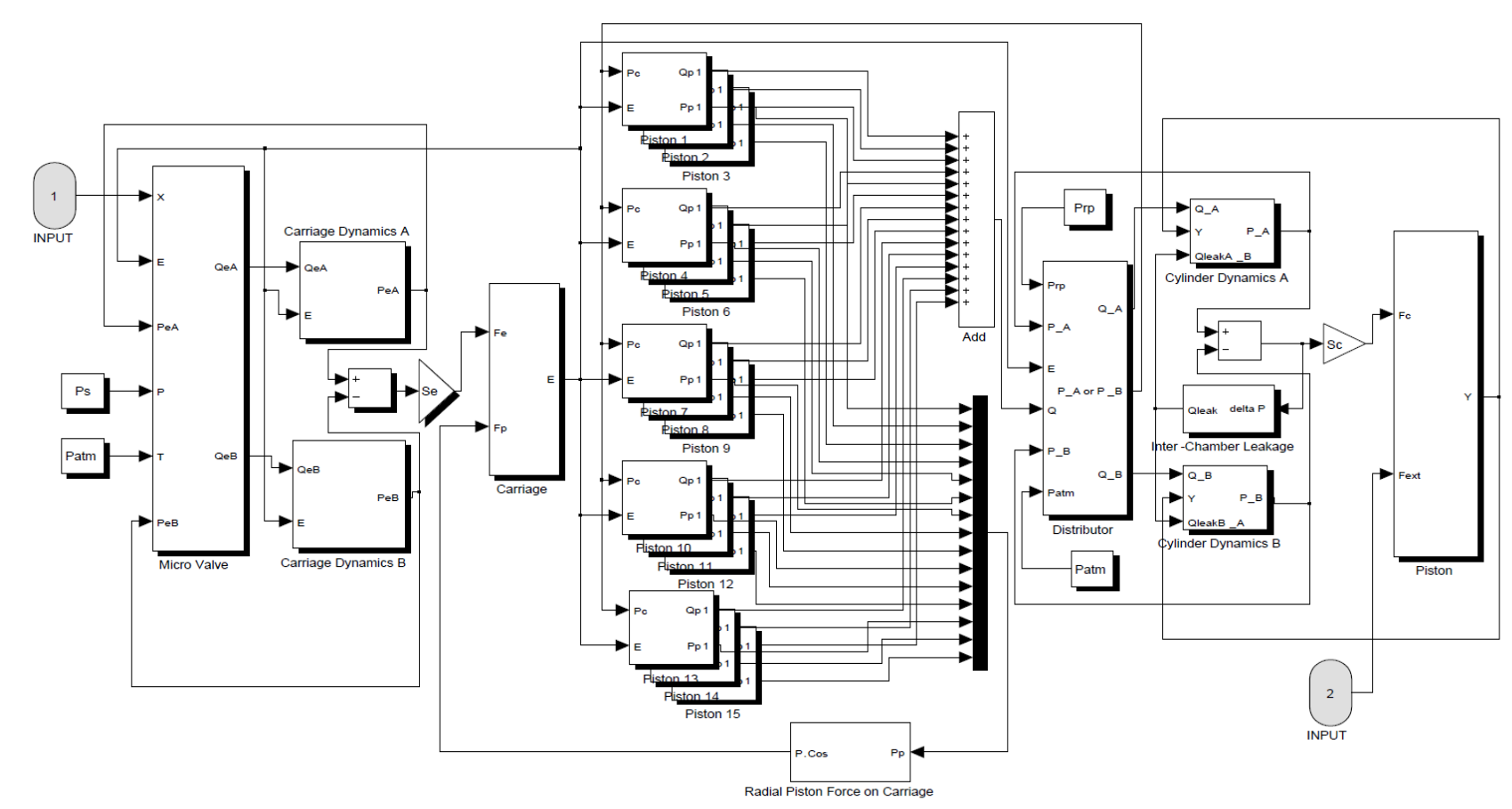

Appendix I: Schematic View of the Virtual Model in Matlab-Simulink. 